\title{
ISOMORPHISMS AND ISOMETRIC MULTIPLIERS
}

\author{
FRANK T. BIRTEL
}

1. Introduction. Let $G$ be a locally compact abelian group with dual group $\dot{G}$. Let $L_{1}(G)$ be the group algebra of $G$ and $M(G)$ be the algebra of bounded measures on $G$. By $M(\dot{G})$ designate the algebra of FourierStieltjes transforms of bounded measures. A necessary and sufficient condition given by Helson [7] for a complex-valued function $f$ on $\dot{G}$ to be an element of $M(\dot{G})$ is that $f \cdot L_{1}(G)^{\frown} \subset L_{1}(G)^{\frown}$. A natural generalization then is to consider the set $A^{m}$ of all complex-valued functions $f$ on the maximal regular ideal space $\mathbb{T}(A)$ of a commutative semi-simple Banach algebra $A$ with the property that $f \cdot \hat{A} \subset \hat{A}$. Banach algebra terminology is in accord with Loomis [8].

Each function $f$ in $A^{m}$ induces a bounded operator $f$ on $A$ given by $(f x)^{-}=f \hat{x} \quad$ for $\quad x \in A$. Let $A^{m}=\left\{f: f \in A^{m}\right\} \quad$ and $^{1} \quad A_{0}^{m}$ $=\left\{f: f \in A^{m} \cap C_{0}(\mathscr{T}(A))\right\} . A^{m}$ and $A_{0}^{m}$ are commutative semi-simple Banach algebras under the uniform operator norm, called, respectively, the algebra of multipliers and the algebra of multipliers vanishing at infinity of $A$. These facts and the basic theory of algebras of multipliers were developed in [1].

From our opening remark, it is clear that if $A=L_{1}(G)$, then $A^{m}$ is isometrically isomorphic to $M(G)$. Quite a few analogues of results about measure algebras are true for abstract algebras of multipliers, even though integration over $G$ is not available in the general setting. Helson, Rudin and Cohen $[7 ; 9 ; 3]$ have proved that an isomorphism between group algebras extends to an isomorphism between the measure algebras and that an isomorphism between measure algebras produces an isomorphism between the underlying group algebras. In $\S 2$, we investigate whether similar propositions obtain between certain Banach algebras and their associated algebras of multipliers. In $\S 3$, under the assumption that the unit ball of $\boldsymbol{A}^{m}$ is weak operator compact, we show that the set of isometric multipliers forms a compact topological group, which can be utilized in a manner analogous to the situation in which there is an underlying group. Specifically, whenever the isometric multipliers separate points of $\mathfrak{N}(A), A$ is spanned by its idempotents and $\mathfrak{T}(A)$ is discrete, reminiscent of certain known facts about group algebras $L_{1}(G)$ for compact $G$. When

Presented to the Society, October 22, 1960; received by the editors September 17, 1960 and, in revised form, March 20, 1961.

${ }^{1} C_{0}(X)$ indicates all continuous functions vanishing at infinity on a locally compact space $X$. 
$A$ is closed in $\boldsymbol{A}^{m}$, these theorems provide particular instances of the applicability of results of $\S 2$.

The suggestions and assistance of Professor Irving Glicksberg are gratefully acknowledged. ${ }^{2}$

2. Isomorphisms of algebras of multipliers. Let $A_{1}$ and $A_{2}$ be commutative semi-simple Banach algebras and let $A_{1}^{m}$ and $A_{2}^{m}$, respectively, be their associated algebras of multipliers. If $T: A_{1} \rightarrow A_{2}$ is an isomorphism of $A_{1}$ onto $A_{2}$, then $T$ induces an adjoint map $\tau: \operatorname{TT}\left(A_{2}\right)$ $\rightarrow \mathfrak{N}\left(A_{1}\right)$ which is a homeomorphism, $\tau$ being given explicitly by the formula:

$$
(T x)^{-}\left(M_{2}\right)=\hat{x}\left(\tau M_{2}\right) \quad \text { where } x \in A \text { and } M_{2} \in \mathfrak{M}\left(A_{2}\right) .
$$

Now $f \in C\left(\mathscr{T}\left(A_{2}\right)\right)$ is in $A_{2}^{m}$ if and only if there exists $g \in A_{1}^{m}$ such that $g\left(\tau M_{2}\right)=f\left(M_{2}\right)$ : Suppose there is a $g \in A_{1}^{m}$ such that $g\left(\tau M_{2}\right)=f\left(M_{2}\right)$. Then $\hat{x} g\left(\tau M_{2}\right)=\hat{x}\left(\tau M_{2}\right) g\left(\tau M_{2}\right)=\hat{x}\left(\tau M_{2}\right) f\left(M_{2}\right)=(T x)^{-}\left(M_{2}\right) f\left(M_{2}\right)$ $=\left((T x)^{-} f\right)\left(M_{2}\right) . T x$ is any element of $A_{2}$. $(T x)^{\frown} f \in A_{2}$, hence $f \in A_{2}^{m}$. And the same argument applies for $T^{-1}$. From this it is clear that $T$ can be extended to an isomorphism of $A_{1}^{m}$ onto $A_{2}^{m}$.

Assume now that $T: A_{1}^{m} \rightarrow A_{2}^{m}$ is an isomorphism and further assume that $A_{1}, A_{2}$ are regular and that ${ }^{3} j_{A_{1}}(\infty), j_{A_{2}}(\infty)$ are respectively dense in $A_{1}$ and $A_{2}$. T induces a homeomorphism $\tau$ of $\mathfrak{T}\left(A_{2}^{m}\right)$ onto $\mathfrak{T}\left(A_{1}^{m}\right)$ as in (1). Let $J_{i}=\left\{f \in A_{i}^{m}: \hat{f}\right.$ vanishes outside some compact subset of $\left.\mathfrak{T}\left(A_{i}\right), i=1,2\right\}$. Let $f_{i} \in J_{i}$ and $f_{i}$ vanish off $C_{i}$. Choose $V_{i}$ a closed neighborhood of infinity in compactified $\mathfrak{M}\left(A_{i}\right)$ such that $V_{i} \cap C_{i}=\varnothing$. Further choose $x_{i} \in A_{i}$ such that $\hat{x}_{i}=0$ on $V_{i}$ and $\hat{x}_{i}=1$ on $C_{i}$. Then $f_{i}=f_{i} \hat{x}_{i} \in \hat{A}_{i}$; indeed, $f_{i} \in j_{A_{i}}(\infty)$. Now were $\tau\left(\mathfrak{T}\left(A_{2}\right)\right)$ $=\mathfrak{M}\left(A_{1}\right), T$ would map $J_{1}$ onto $J_{2}$; and, were $A_{i}$ closed in $A_{i}^{m}, T$ would have to map $A_{1}$ onto $A_{2}$, because $j_{A_{i}}(\infty)$ and $j_{A_{2}}(\infty)$ are dense.

$\tau\left(\mathfrak{T}\left(A_{2}\right)\right)=\mathfrak{T}\left(A_{1}\right)$ when we restrict the discussion of the last paragraph to the algebras $A_{1_{0}}^{m}, A_{2_{0}}^{m}$, of multipliers vanishing at infinity, since in this case $\mathfrak{T}\left(A_{1}\right)$ and $\mathfrak{N}\left(A_{2}\right)$ by regularity are the unique minimal closed sets such that multipliers vanishing thereon vanish identically.

Methods employed in the above discussion are essentially those of Helson [7] and Rudin [9], modified to apply to the more general setting. If $A=L_{1}(G), G$ locally compact, then $j(\infty)$ is dense in $A$ and $A$ is a closed ideal in $M(G)$.

2 This is a portion of the author's dissertation submitted to the University of Notre Dame, June, 1960.

${ }^{3} j_{A}(\infty)$ is the set of all $x \in A$ such that $\hat{x}$ has compact support in $\operatorname{Mr}(A)$. For regular $A$, if $j_{A}(\infty)^{-}=A$, then $A$ is said to be tauberian.

- For the relation of $\mathfrak{M}\left(A^{\mathrm{m}}\right)$ and $\mathfrak{M}(A)$, see [1]. 
Summarizing, we have

THEOREM 1. An isomorphism of two commutative semi-simple Banach algebras can be extended to an isomorphism of their associated algebras of multipliers. An isomorphism of the algebras of multipliers vanishing at infinity of two regular tauberian commutative semi-simple Banach algebras $A_{1}, A_{2}$ induces an isomorphism of $A_{1}$ and $A_{2}$, provided that both are closed in their respective algebras of multipliers.

That Theorem 1 is not true for arbitrary full algebras of multipliers can be seen from the following example: if $D$ is the algebra of continuous functions on $\{z:|z| \leqq 1\}$, analytic on $\{z:|z|<1\}$, and $A=z D$, then $A^{m}=D$, and we can get an automorphism of $A^{m}$ by sending $f \rightarrow f \circ \tau$ with $\tau(z)=(z-a) /(1-\bar{a} z) \quad(|a|<1)$. But $A$ is not mapped on $A$, nor $\mathfrak{T H}(A)$ on $\mathfrak{T}(A)$.

However, under certain conditions, an automorphism $T$ of the full algebra $A^{m}$ of multipliers of $A$ does induce an automorphism of the underlying algebra $A$.

Theorem 2. Let $A$ be a commutative semi-simple Banach algebra with discrete maximal regular ideal space $\operatorname{m(}(A)$. If $A$ is generated by its idempotents and closed in $A^{m}$, then every automorphism $T$ of $A^{m}$ induces an automorphism of $A$.

Proof. It is clear that $\operatorname{TM}(A)$ is discrete in $\operatorname{Tr}\left(A^{m}\right)$. Suppose $M \in \mathfrak{M}\left(A^{m}\right) \backslash \mathfrak{M}(A)$. If $\{M\}$ is open-and-closed, then, by a wellknown theorem of Silov (see [10]), there exists $f \in A^{m}$ such that $\hat{f}$ is the characteristic function of the set $\{M\}$. But this is impossible, since $\hat{f}$ would vanish on $\mathfrak{T}(A)$ and hence must vanish on all of $\mathscr{T}\left(A^{m}\right)$. Consequently no point of $\mathfrak{N}\left(A^{m}\right) \backslash \mathfrak{T}(A)$ is isolated.

$T$ induces a homeomorphism $\tau$ of $\mathfrak{N}\left(A^{m}\right)$ onto itself given by $\hat{f}(\tau M)=(T f)-(M)$ for $M \in \mathfrak{N}\left(A^{m}\right) . \tau$ takes isolated points into isolated points, so $\tau(\mathfrak{T}(A))=\mathfrak{T}(A)$.

Since $\operatorname{Tl}(A)$ is discrete, again by Silov's theorem, $A$ is regular. The closed span of the idempotents of $A$ is $j_{A}(\infty)$. But every $f \in A^{m}$ which vanishes off a compact (finite) subset of $\mathfrak{T}(A)$ is clearly in $A$ and, in particular, in $j_{A}(\infty) . T$ maps $j_{A}(\infty)$ into $j_{A}(\infty)$, since $\tau(\mathfrak{T}(A))$ $=\mathfrak{T}(A)$. Therefore, $T$ maps $A$ onto $A$.

3. Isometric multipliers. In some sense restrictions imposed on the isometric onto multipliers of $A$ determine structural properties of $A$ very much similar to properties of group algebras (e.g. see [12]). The restriction which we wish to impose is that the closed unit ball of $\boldsymbol{A}^{\boldsymbol{m}}$ be compact in the weak operator topology, the weakest topology rendering all maps $T \rightarrow\left\langle T x, x^{*}\right\rangle, x \in A, x^{*} \in A^{*}, T$ a bounded operator on $A$, continuous. 
TheOREM 3. Let $\mathbf{G}$ be the set of isometric multipliers mapping $A$ onto $A$. If the closed unit ball of $A^{m}$ is compact in the weak operator topology, then $\mathrm{G}$ is a compact topological group.

Proof. It is clear that $G$ is a topological semi-group, since under the weak operator topology multiplication is separately continuous. We will first show that $G$ is a group which is weak operator closed in the unit ball of $\boldsymbol{A}^{m}$ and hence compact.

The inverse of an onto isometry is of course an onto isometry and the identity

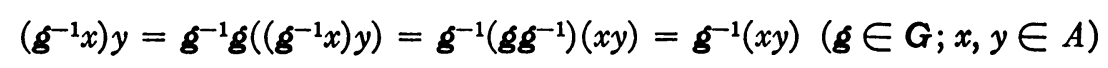

shows that $\mathscr{g}^{-1} \in A^{m}$. See [1, Theorem 4]. Thus $G$ is a group. Any element $f$ in the closure of $G$ is of course in $A^{m}$, but it remains to show that $\boldsymbol{f}$ is an isometry. Let $\left\{\boldsymbol{g}_{\delta}\right\}$ be a net in $G$ converging to $f$. Then, in particular, $\rho\left(\delta_{\delta} x\right) \rightarrow \rho(f x)$ where $\rho$ is a multiplicative functional on $A$. By results of $[1],\left|g_{\delta}(\rho)\right|^{n} \leqq\left\|\xi_{\delta}\right\|=1$ for all integers $n$, so $\left|g_{\delta}(\rho)\right|=1$ and $|f(\rho)| \leqq\|f\|$, so $\|f\|=1$. Now let $h$ be an adherent point of the net $\left\{\boldsymbol{g}^{-1}\right\}$. There is a sub-net $\left\{\boldsymbol{g}_{\mu}^{-1}\right\}$ of $\left\{\boldsymbol{g}^{-1}\right\}$ which converges to $h$ and

$$
\begin{aligned}
|1-h(\rho) f(\rho)| & \leqq\left|g_{\mu}(\rho)-f(\rho)\right|\left|g_{\mu}^{-1}(\rho)\right|+\left|g_{\mu}^{-1}(\rho)-h(\rho)\right||f(\rho)| \\
& \leqq\left|g_{\mu}(\rho)-f(\rho)\right|+\left|g_{\mu}^{-1}(\rho)-f(\rho)\right| \rightarrow 0 .
\end{aligned}
$$

Therefore $h$ and $f$ as functions on $\mathfrak{T}(A)$ are inverses and $h f$ or $f h$ determine the identity operator on $A$.

Since $f$ and $h$ are of norm $\leqq 1$, we conclude that each is an isometry, whence $f \in G$ and $G$ is closed, so compact.

And by a known result of Ellis [6], a compact topological semigroup which is also a group is a compact topological group (joint continuity of multiplication). The theorem is proved.

Let $d \boldsymbol{g}$ denote normalized Haar measure on $\boldsymbol{G}$. If $f: G \rightarrow A$ is a continuous vector-valued function from $G$ into $A$ then by

$$
\int f(\boldsymbol{g}) d \boldsymbol{g}
$$

we shall mean the unique element $x$ of $A$ such that

$$
\left\langle x, x^{*}\right\rangle=\int_{G}\left\langle f(\boldsymbol{g}), x^{*}\right\rangle d \boldsymbol{g}, \quad \text { all } x^{*} \in A^{*} .
$$

The existence of (2) being guaranteed (see [2]) by the fact that the weakly closed convex hull of a weakly compact subset of $A$ is weakly 
compact (see [5]). Also there is defined on $\boldsymbol{G}$ an approximate identity $\left\{\phi_{\sigma}\right\}$ composed of trigonometric polynomials [11] and

$$
\lim _{\boldsymbol{\sigma}} \int_{\boldsymbol{G}} \boldsymbol{\phi}_{\boldsymbol{\sigma}}(\boldsymbol{\xi}) f(\boldsymbol{\xi}) d \boldsymbol{g}=f(\boldsymbol{\theta})
$$

where $e$ is the identity of $G$.

THEOREM 4. $A$ is strongly spanned by the common eigenvectors of $G$ which have eigenvalues of modulus 1 .

PRoof. $\boldsymbol{g} \rightarrow \boldsymbol{g} x$, for fixed $x \in A$, is a continuous function from $G$ in the weak operator topology into $A$ in its weak topology. Take $\left\{\phi_{\sigma}\right\}$ to be an approximate identity of trigonometric polynomials of $G$ and define

$$
\boldsymbol{f}_{\sigma} x=\int_{G} \phi_{\sigma}(\boldsymbol{g}) \boldsymbol{g} x d \boldsymbol{g}
$$

$f_{\sigma} x$ converges to $e x=x$. Let $\dot{\boldsymbol{g}} \in \dot{G}$ and $\langle\cdot, \cdot \cdot\rangle$ indicate the usual pairing between $G$ and $\dot{G}$. For $\xi_{0} \in G$, we have

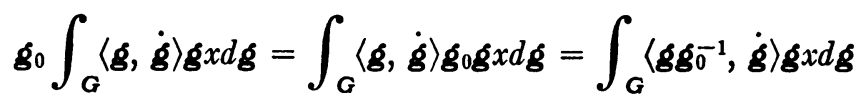

$$
\begin{aligned}
& =\langle\overline{\dot{\mathbf{g}}, \boldsymbol{g}}\rangle \int_{G}\langle\boldsymbol{g}, \dot{\mathbf{g}}\rangle \boldsymbol{g} x d \dot{\mathbf{g}} \text {. }
\end{aligned}
$$

Hence setting

$$
y=\int_{\mathbf{G}}\langle\boldsymbol{\xi}, \dot{\boldsymbol{g}}\rangle \boldsymbol{g} x d \boldsymbol{\mathcal { B }}, \text { we have } \boldsymbol{g}_{0} y=\left\langle\overline{\boldsymbol{g}_{0}, \dot{\boldsymbol{g}}}\right\rangle,
$$

so $y$ is a common eigenvector of $G$ with eigenvalue of modulus 1 . Therefore using (3), we see that the weakly closed span of these common eigenvectors is $A$. And by Mazur's theorem we ascertain that $A$ is actually the strongly closed span of these common eigenvectors.

Lemma. If $G$ separaies the points of $\mathfrak{N}(A)$ and $x$ is a common eigenvector of $G$ with eigenvalue of modulus 1 , then $\hat{x}$ has one-point support.

Proof. $\boldsymbol{g} x=\lambda_{\theta} x$ and $(\boldsymbol{\xi} x)^{\wedge}=\lambda_{\theta} \hat{x}$, so $g(M)=\lambda_{\theta}$ for all $M \in \mathfrak{M r}(A)$ such that $\hat{x}(M) \neq 0$. Suppose $\hat{x}\left(M_{1}\right) \neq 0 \neq \hat{x}\left(M_{2}\right), M_{1} \neq M_{2}$, and $M_{1}, M_{2}$ $\in \mathscr{M}(A)$. Then

$$
g\left(M_{1}\right) \hat{x}\left(M_{1}\right)=\lambda_{v} \hat{x}\left(M_{1}\right) ; \quad g\left(M_{2}\right) \hat{x}\left(M_{2}\right)=\lambda_{\theta} \hat{x}\left(M_{2}\right) .
$$

Hence $g\left(M_{1}\right)=g\left(M_{2}\right)=\lambda_{\theta}$, contradicting the fact that $G$ separates the 
points of $\mathfrak{T}(A)$. Therefore any common eigenvector with eigenvalue of modulus 1 has one-point support.

THEOREM 5. If $G$ separates points of $\operatorname{Tr}(A)$ and the unit ball of $A^{m}$ is compact in the weak operator topology, then $\operatorname{Ml}(A)$ is discrete and $A$ is spanned by its idempotents.

Proof. Any $x \in A$ such that $\hat{x}$ has one-point support is a common eigenvector of $G$, indeed of $\boldsymbol{A}^{m}$. By the preceding lemma it is clear that the set $E$ of all common eigenvectors $x$, such that $\hat{x}=0$ on $\mathfrak{M}(A) \backslash\{M\}$ for some $M \in \mathscr{M}(A)$ and $\hat{x}(M)=1$, spans $A . A$ is consequently spanned by its idempotents. Furthermore, not all $x \in E$ vanish at any point $M \in \mathscr{T}(A)$, since $A$ separates points of $\mathscr{N}(A)$. Therefore for each $M \in \mathfrak{N}(A)$ there exists $x \in E$ such that $\hat{x}(M)=1$ and $\left\{M^{\prime}:\left|\hat{x}\left(M^{\prime}\right)-\hat{x}(M)\right|=\left|\hat{x}\left(M^{\prime}\right)-1\right|<1 / 2\right\}$ is a neighborhood of $M$. So $\mathfrak{M}(A)$ is discrete.

We conclude by pointing out the strong analogy of the above theorem with certain properties of group algebras which was alluded to earlier. Let $H$ be a compact group and consider $A=L_{1}(H)$. For $T \in L_{1}(H)^{m}$ it is known that there exists $m \in M(H)$ such that $T f$ $=f * m$ for all $f \in L_{1}(H)$. The correspondence $T \rightarrow m$ is an isometric isomorphism of $L_{1}(H)^{m}$ onto $M(H)$. Furthermore $(f * m, h)=(f * h, m)$ for $h \in L_{\infty}(H) . f * h \in C(H)$. Hence the weak operator topology and the weak* topology coincide on bounded sets. $M(H)=C(H)^{*}$, and therefore, in this case as above, the strongly closed unit ball of $L_{1}(H)^{m}$ is weak operator compact; $G$, the group of isometric onto multipliers in the unit ball of $L_{1}(H)^{m}$, is the group of unimodular multiples of point masses. And of course, $\mathfrak{T}\left(L_{1}(H)\right)=\dot{H}$ is discrete.

\section{REFERENCES}

1. F. T. Birtel, Banach algebras of multipliers, Duke Math. J. 28 (1961), 203-212.

2. N. Bourbaki, Intégration. Eléments de mathématique. XIII. Book VI, Actualités Sci. Ind. no. 1175, Hermann, Paris, 1952.

3. P. J. Cohen, Homomorphisms and idempotents of group algebras, Bull. Amer. Math. Soc. 65 (1959), 120-123.

4. W. F. Eberlein, Characterizations of Fourier-Stieltjes transforms, Duke Math. J. 22 (1955), 465-468.

5. - Abstract ergodic theorems and weakly almost periodic functions, Michigan Math. J. 3 (1955-1956), 137-139. 126.

6. R. Ellis, Locally compact transformation groups, Duke Math. J. 24 (1957), 119-

7. E. Helson, Isomorphisms of abelian group algebras, Ark. Math. 2 (1953), 475487.

8. L. H. Loomis, An introduction to abstract harmonic analysis, Van Nostrand, New York, 1953. 
9. W. Rudin, Measure algebras on abelian groups, Bull. Amer. Math. Soc. 65 (1959), 227-247.

10. G. E. Silov, On decomposition of a commutative normed ring in a direct sum of ideals, Mat. Sb. 32 (1953), 353-364.

11. A. Weil, L'intégration dans les groupes topologiques et ses applications, Actualités Sci. Ind. no. 869, Hermann, Paris, 1940.

12. J. Wermer, Ideals in a class of commutative Banach algebras, Duke Math. J. 20 (1953), 273-278.

Ohio State University and

UNiversity of Notre DaMe

\section{A MULTI-POINT GENERALIZATION OF NEWTON'S DIVIDED DIFFERENCE FORMULA}

\section{HERBERT E. SALZER}

Newton's divided difference formula for the polynomial of degree $n-1$ determined by the values of the function $f(x)$ and its divided differences up to the $(n-1)$ th order originating from any single point, has the following multi-point generalization:

Theorem I. The unique interpolation polynomial $P(x)$, of degree $\sum_{i=1}^{n} r_{i}-1$, which is determined by $f\left(x_{i}\right) \equiv f_{i} \equiv D^{0} f_{i}$ and the first $r_{i}-1$ divided differences, $\mathfrak{D}^{m} f_{i} \equiv\left[x_{i} x_{i_{1}} \cdots x_{i_{m}}\right], m=1, \cdots, r_{i}-1$, originating from the $n$ points $x_{i}, i=1, \cdots, n$, is expressible as

(1) $\quad P(x)=\sum_{i=1}^{n} \Pi_{i}(x) \psi_{i}(x)$, where

(2) $\quad \Pi_{i}(x)=\prod_{j=1, j \neq i}^{n}\left(x-x_{j}\right)\left(x-x_{j_{1}}\right) \cdots\left(x-x_{j_{r_{j}-1}}\right)$,

(3) $\quad \psi_{i}(x)=\sum_{m=0}^{r_{i}-1}\left[x_{i} x_{i_{1}} \cdots x_{i_{m}}\right] \psi_{i, m}(x)$, and

(4) $\psi_{i, m}(x)=\sum_{s=0}^{r_{i}-m-1}\left[x_{i_{m}} x_{i_{m+1}} \cdots x_{i_{m+}}\right]_{1 / \mathbb{I}_{i}}\left(x-x_{i}\right)\left(x-x_{i_{1}}\right) \cdots$

$$
\left(x-x_{i_{m+1-1}}\right) .
$$

In (3) and (4), $x_{i_{0}} \equiv x_{i} ;$ in (3), $\left[x_{i}\right] \equiv f_{i} ;$ in (4), $\left[x_{i_{m}}\right]_{1 / \Pi_{i}} \equiv 1 / \Pi_{i}\left(x_{i_{m}}\right)$, the sth order divided difference $\left[x_{i_{m}} x_{i_{m+1}} \cdots x_{i_{m+l}}\right]_{1 / \mathrm{I}_{i}}$ is for the function $1 / \Pi_{i}(x)$, and $\psi_{i, 0}(x)$ begins $\left[x_{i}\right]_{1 / \Pi_{i}}+\cdots$.

Presented to the Society, April 22, 1961; received by the editors February 23, 1961. 